\title{
SHAPE OPTIMIZATION OF ROAD TUNNEL CROSS-SECTION BY SIMULATED ANNEALING
}

\author{
MACIEJ SOBÓTKA, Michat PACHNICZ \\ Faculty of Civil Engineering, Wrocław University of Science and Technology, \\ Wybrzeże Wyspiańskiego 27, 50-370 Wrocław, Poland, \\ e-mail: maciej.sobotka@pwr.edu.pl,michal.pachnicz@pwr.edu.pl
}

\begin{abstract}
The paper concerns shape optimization of a tunnel excavation cross-section. The study incorporates optimization procedure of the simulated annealing (SA). The form of a cost function derives from the energetic optimality condition, formulated in the authors' previous papers. The utilized algorithm takes advantage of the optimization procedure already published by the authors. Unlike other approaches presented in literature, the one introduced in this paper takes into consideration a practical requirement of preserving fixed clearance gauge. Itasca Flac software is utilized in numerical examples. The optimal excavation shapes are determined for five different in situ stress ratios. This factor significantly affects the optimal topology of excavation. The resulting shapes are elongated in the direction of a principal stress greater value. Moreover, the obtained optimal shapes have smooth contours circumscribing the gauge.
\end{abstract}

Key words: simulated annealing, underground excavation, shape optimization, linear elasticity, energy of volumetric strain

\section{INTRODUCTION}

Size, shape and topology optimization methods give a possibility to reduce costs of the construction and to provide favourable conditions of structure operation. The optimization in tunnelling engineering concerns mainly the opening shape [1]-[4] but also the other elements of a design like arrangement of rock bolts [5]. The first aspect, however, is the most vital element of the design.

The pioneering works dealing with the topic of underground excavation shape optimization appeared in literature in the 1960s (e.g., [6]). The optimal shape was determined by specifying the shape class and the proper ratio of opening sizes dependent on the horizontal and vertical principal stress ratio. The theoretical considerations [6] are based on a solution of a plain problem of stress distribution around the elliptical opening in linear elastic material. Thus, the ellipse has been a priori assumed to be the optimal shape. Taking this into consideration, the only unknown to be determined is the scalar value of semi-axes ratio. It can be quite easily proven that for unsupported tunnel excavation the optimal ratio of ellipse sizes, providing the minimum value of the compressive normal stress occurring within the rock mass, is equal to the ratio of the virgin stresses [3], [6]

$$
m_{\mathrm{opt}}=\left(\frac{a}{b}\right)_{\mathrm{opt}}=\frac{p_{x}}{p_{y}}
$$

where $m_{\mathrm{opt}}$ - optimal ratio of the semi-axes of an ellipse, $a, b$ - lengths of semi-axes, horizontal and vertical, respectively, $p_{x}$ and $p_{y}$ - in situ normal stresses, horizontal and vertical, respectively.

For the excavation with a structural support the optimal ratio of ellipse semi-axes is equal to the square root of in situ stresses ratio

$$
m_{\mathrm{opt}}=\left(\frac{a}{b}\right)_{\mathrm{opt}}=\sqrt{\frac{p_{x}}{p_{z}}} .
$$

This condition should provide minimum effort of the support material [1], [4], [6].

In 2005, Ren et al. [2] used the evolutionary structural optimization (ESO) procedure [7] [8] to solve the underground excavation optimization problem. The obtained results confirm the hypotheses formulated for unsupported tunnel excavations, i.e., the obtained shapes are ellipse like and the ratio of horizontal to vertical dimensions agrees with equation (1).

In 2009, the authors of the present work formulated an energetic optimization condition for tunnel excavation with a structural support [3]. The verification of the proposed condition has shown the agreement with formula (2) [1]. Furthermore, in [4] a heu- 
ristic, stochastic method called simulated annealing (SA) [4], [9]-[13] with the energetic condition [1], [3], [4], [11] as a cost function has been applied to find optimal shapes of excavation. The results obtained justify the assumption that the optimal shape is actually an ellipse. In the aforementioned work [4] the fixed value of the tunnel cross-section area has been assumed. Such a simple approach has enabled some theoretical hypotheses to be proved, but it does not meet the practical design requirements. In the engineering practice, the assumption of fixed area should be replaced by the following: excavation cross-section must contain a clearance gauge specified in the design. In some of the tunnelling methods such as TBM the shape type is determined as circular. In such a case, knowing the shape of a clearance gauge, determination of excavation size (diameter) is straightforward - the circular liner must circumscribe the gauge. In the other methods, like NATM, a tunnel cross-section can be shaped relatively freely. In such a situation, only some qualitative general rules are utilized. For instance, the shape of a liner in NATM must be smooth to avoid stress concentration, i.e., sharp bends should be avoided [14], [15]. Such a guideline does not give an exact solution for shaping the section.

Recapitulating, most of the theoretical approaches are too formal and, therefore, do not meet the realistic design requirements whereas the practical rules do not guarantee obtaining the exact, conclusive solution. The paper proposes a methodology that eliminates the above-mentioned disadvantages of both approaches. The work takes advantage of the procedure given in [4] and [11] modified so that it allows us to utilize the condition of preserving a defined clearance gauge.

The arrangement of the work is as follows. First, the formulation of energetic optimality condition is outlined. Next, the general concept of simulated annealing procedure is briefly explained. Subsequently, the proposed optimization procedure is presented. This is followed by the numerical examples. The optimal shapes of excavation are determined for five different values of the in situ stress ratios. A discussion of the results and conclusions end the paper.

\section{ENERGETIC OPTIMALITY CONDITION}

A crucial assumption, adopted in the formulation of the energetic optimality condition [1], [3], [4], is that the primary task of a liner support is retaining the excavation convergence. Thus, the best shape of a tunnel cross-section is the one that minimizes the convergence. Accounting this, a cost function must be a measure of the convergence. It is postulated as a hypothetic energy of volumetric strain cumulated in the excavation core

$$
E_{0}=\frac{1}{2} K \int_{\Omega}\left(\varepsilon_{x}+\varepsilon_{y}\right) d \Omega,
$$

where $K$ denotes the bulk modulus of the rock mass, $\varepsilon_{x}, \varepsilon_{y}$ are the values of normal strain components, horizontal and vertical, respectively, $\Omega$ is the area of the excavation cross-section.

Having regard to the above mentioned definition of the cost function, it is convenient to consider the area of excavation, which is in fact a void, as a very compliant material. Such a manner is suitable especially in the numerical formulation when iterative optimization methods are involved. There is no need, then, for changing geometry and boundary conditions during the procedure. Furthermore, no support is taken into account within this methodology. Therefore, no properties of the support have to be assumed.

\section{SIMULATED ANNEALING}

Simulated annealing [9] is a metaheuristic, stochastic method of optimization, utilized to approximate global optimum in a large space, e.g., in shape and topology optimization problems in structural design [4], [10], [11], [16]. The procedure derives from a physical process in metallurgy, called annealing. It is used to improve the microstructure of metal alloys. The annealing consists of a few stages. Firstly, the material is heated to above its critical temperature. Then, the temperature is being maintained for a certain time period. The next stage is the crucial one and involves cooling at suitably slow rate. Initially, at high temperatures, the microstructure is being continuously rearranged. The random motion of the particles is governed by the laws of statistical thermodynamics. With decreasing temperature, the microstructure is gradually refined. Eventually, the particles set at low energy levels and the system reaches a stable state, optimal in sense of the value of internal energy.

The SA algorithm takes advantage of the procedure for simulation of a collection of atoms, proposed by Metropolis [17]. At a given temperature, the system is brought to an equilibrium in the following iterative procedure. In each iteration, a randomly chosen atom is given a random displacement and the internal energy of the system is calculated. If the energy in the 
new configuration is less than that of the previous one $(\Delta E<0)$, then the change is always accepted, otherwise the change is accepted with the probability

$$
P(\Delta E)=e^{-\frac{\Delta E}{k_{B} T}},
$$

where $k_{B}$ is the Boltzmann constant and $T$ is the temperature.

An analogous procedure is adopted in the optimization method of simulated annealing. The internal energy is replaced by a proper cost function. The subject of the optimization is gradually refined in the iterative procedure. Random modifications imposed on the system considered are tested and accepted or refused according to the suitable conditions. At a given temperature a certain number of iterations is executed. Then, the temperature is slightly decreased and, again, a certain number of iterations is executed, etc. The procedure is continued until the further decreasing of the temperature gives no effect on the value of the cost function.

As may be noted, the temperature in simulated annealing becomes an artificial parameter, governing the probability of acceptance of unfavourable system modifications. A permission to accept certain number of unfavourable modifications prevents stopping the procedure at local minima. On the other hand, increasing the temperature to suitably low values enables the optimized system to be brought to a stable state in the end of the optimization procedure.

\section{NUMERICAL PROCEDURE FOR SHAPE OPTIMIZATION}

Optimization procedure relies on finding excavation shape, understood as excavation material distribution within the existing grid that provides minimal elastic volumetric strain energy in the area considered.

The numerical procedure for optimization of a tunnel cross-section shape is based on the simulated annealing application [4], [11] with energetic optimality condition [1], [3], [4]. A commercially available finite volume code of Itasca Flac 7.00 [16] has been utilized to solve a series of boundary value problems. The procedure has been written in Fish programming language within Flac package.

The system under consideration is a plain strain elastic deformation problem, being a simplified model of a tunnel excavation in a rock mass. A static scheme of the problem is shown in Fig. 1. The model represents only a quarter of the area around the excavation due to the symmetry.

The domain is a square of a side length of about $40 \mathrm{~m}$. It consists of two complementary subareas - the rock mass $\Gamma$ and the excavation $\Omega$. A rectangular starting shape of excavation $\Omega$ (to be then optimized) is indicated by color filling (see Fig. 1). Within the excavation $\Omega$ there is a clearance gauge of a size

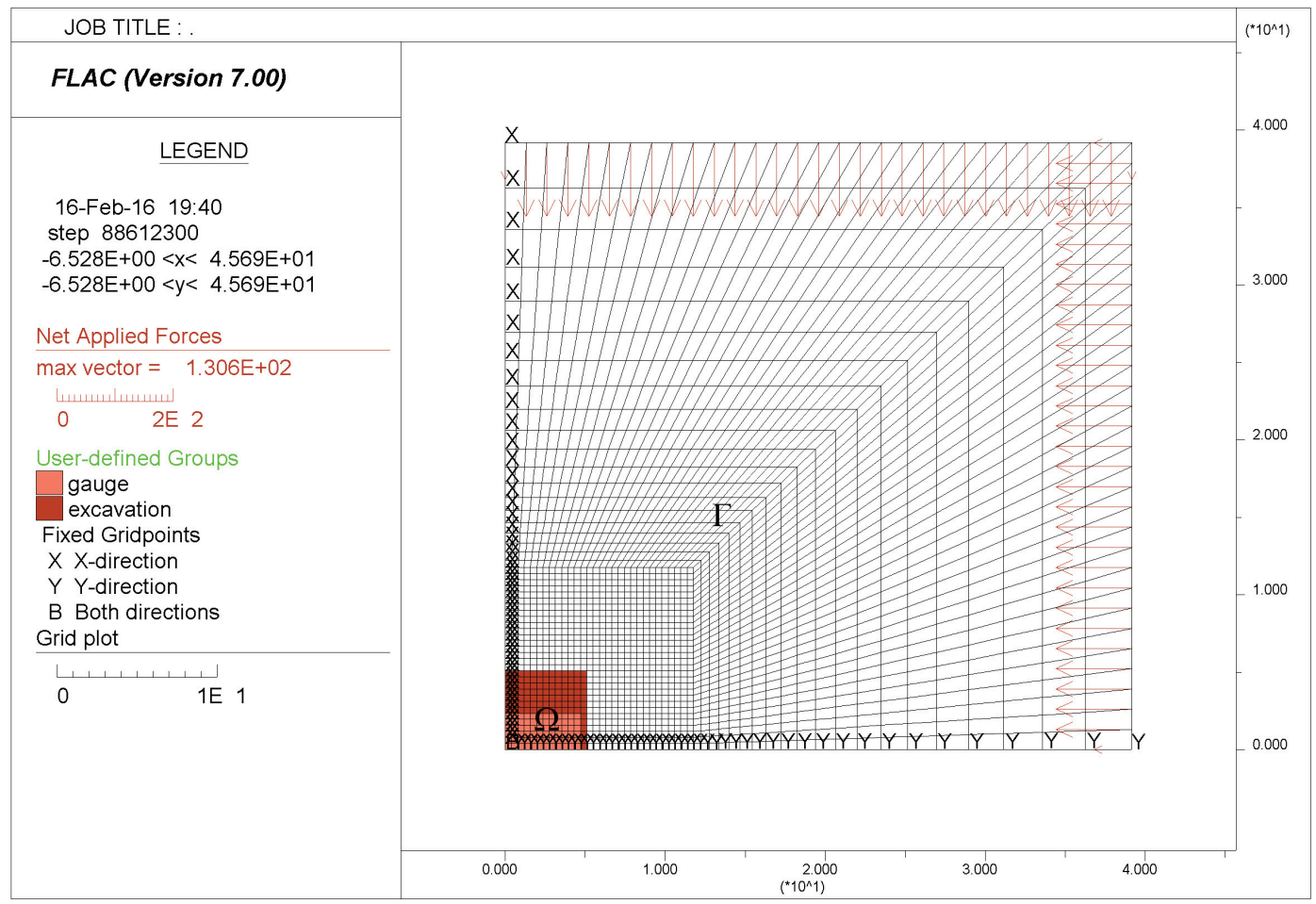

Fig. 1. A boundary value problem under consideration 
$9.4 \mathrm{~m} \times 4.7 \mathrm{~m}$, which is equivalent to $12 \times 6$ cells (lighter colour). This corresponds to the clearance of double lane two-way road with proper margins, e.g., [19]. It is required as a geometry constraint that the entire clearance gauge must be included in the excavation area $\Omega$. The other geometric constraints imposed on the shape are the following. The excavation cannot protrude the area of regular square grid, which is $30 \times 30$ cells (see Fig. 1 ). Both regions, i.e., the rock mass $\Gamma$ and the excavation $\Omega$ must remain simply connected during the optimization procedure. In other words, the regions must never divide into two or more separate regions and must not have the "islands" of the rock mass region, (see the lighter color in Fig. 1 and all the following).

Boundary conditions are adopted as follows: the displacement components perpendicular to edges on the symmetry lines are fixed at the value of 0 . On the other two segments of the boundary the uniform normal stresses equal to in situ stress components are assumed. In the particular, example (Fig. 1), the values of both, the horizontal and vertical virgin stress components are assumed to be equal. It follows that in situ stress ratio is $p_{x} / p_{y}=1.0$. The elasticity parameters of the rock mass are as follows: bulk modulus $K_{\Gamma}=1.0 \mathrm{e} 8 \mathrm{~Pa}$ and shear modulus $G_{\Gamma}=1.0 \mathrm{e} 8 \mathrm{~Pa}$. The parameters adopted for the region of excavation are $K_{\Omega}=1.0 \mathrm{e} 2 \mathrm{~Pa}$ and $G_{\Omega}=1.0 \mathrm{e} 2 \mathrm{~Pa}$.

Within the proposed procedure a single permissible change of shape is considered as a change of region and its parameters $(\Gamma$ or $\Omega$ ) of a chosen cell. Thus, there are two types of them (Fig. 2).

First, a possibility of "enlarging" the excavation is considered. A list of elements belonging to the rock mass that can be switched to excavation region without violating the geometric constraints is generated.
Then, one of those elements is randomly picked and its parameters are changed to excavation parameters. The modified boundary problem is then solved and the value $E_{0}$ is calculated. The change is accepted or rejected according to the conditions given in Section 3. Immediately after that the possibility of excavation "contraction" is considered in the analogous manner. A list of elements from the excavation that can be switched to the rock mass without violating the geometric constraints is generated. Then, one of these elements is randomly chosen and its parameters are changed to rock mass parameters. The boundary problem is solved again and the corresponding energy $E_{0}$ is calculated. Again, the change is accepted or rejected on the basis of the value of the energy increment. The two types of change in shape are executed alternately. As the acceptance of each is considered separately it follows that the size of excavation area may change during the optimization process.

Each iteration of the procedure contains (in order):

- random change in excavation shape,

- solving boundary value problem and calculating cost function,

- acceptance or rejection of the change according to conditions stated in Section 3.

At a given temperature a fixed number of iteration steps $k=200$ are executed.

A temperature drop is performed as the geometric sequence, i.e., a new temperature is 0.9 times the previous one. For succeeding temperature values $T_{i}=T_{i-}$ ${ }_{1} \cdot 0.9$, a number of $k$ iterations is executed and then temperature drop proceeds. The procedure of optimization ends when further decrease in temperature does not cause any change in the cost function value. It follows that in the particular case no further changes in shape occur.
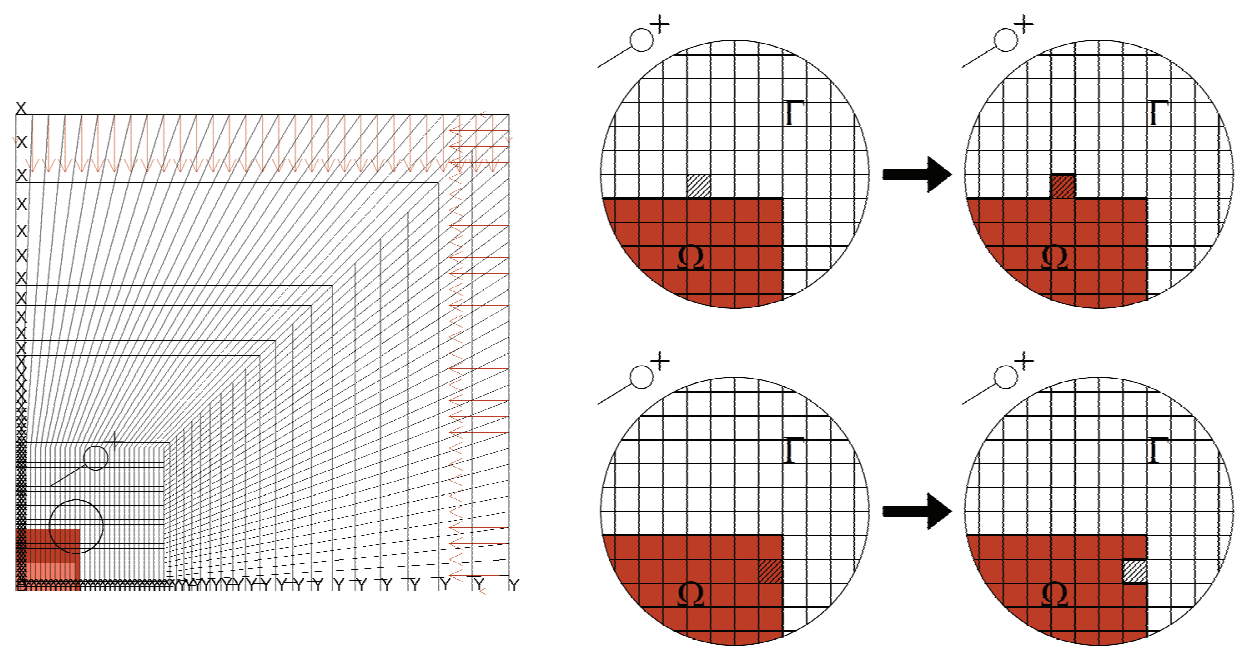

Fig. 2. Two types of permissible changes in shape 
The only aspect that needs clarification is the method for determining a starting temperature $T_{0}$. It is, in general, established in such a way that implies the estimated probability of acceptance of an unfavourable change equal to 0.5 [4], [11] at the beginning of the procedure. First, a series of $n=2000$ changes of a starting shape are proceeded and the average $\overline{\Delta E_{0}}$ of the total number of the positive increments in the energy is calculated. Then the value of $T_{0}$ is derived from equation (4) by applying the substitutions: $P=$ $0.5, \Delta E_{0}=\overline{\Delta E_{0}}, T=T_{0}$. Finally, $T_{0}$ can be obtained from the following expression

$$
T_{0}=-\frac{\overline{\Delta E_{0}}}{\ln 0.5}
$$

\section{NUMERICAL EXAMPLES}

The evolution of the shape in the case considered $\left(p_{x} / p_{y}=1\right)$ is presented in Fig. 3. The initial shape and shapes after 2000, 4000, 10000 and 20000 iterations are shown. For better legibility of the results the plots are limited to the regular $30 \times 30$ cells grid.

The procedure outlined in the previous section has been carried out for five different ratios of in situ stress, namely $p_{x} / p_{z}=1 / 4, p_{x} / p_{z}=1 / 2, p_{x} / p_{z}=1, p_{x} / p_{z}=2$ and $p_{x} / p_{z}=4$. The obtained optimal shapes are presented in Fig. 4.

In all the cases of in situ stress the shape stabilizes after approximately ten thousand iterations, which corresponds to final temperature $T=0.9^{50} T_{0}$. a)

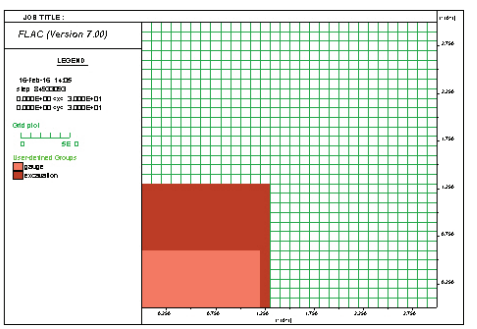

b)

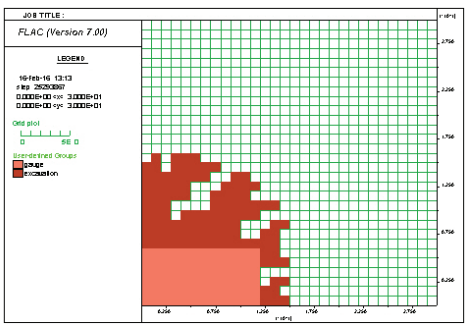

c)

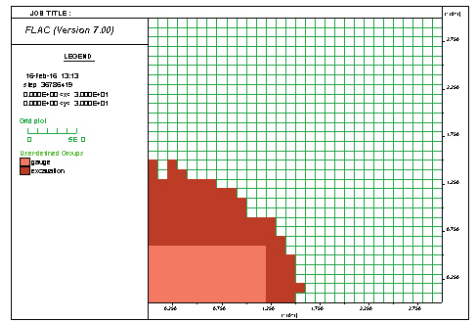

d)

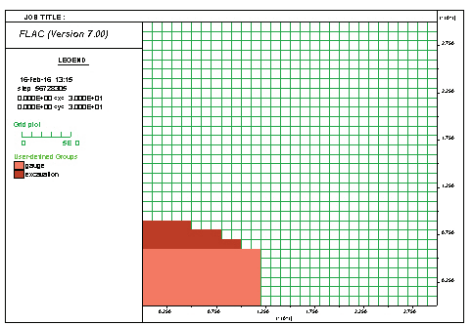

e)

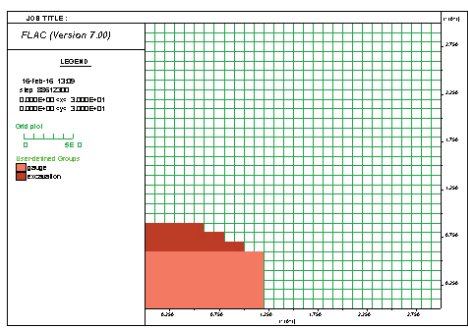

Fig. 3. Evolution of the shape of excavation during the simulated annealing procedure: (a) the initial shape; (b), (c), (d) and (e) - shapes after 2000, 4000, 10000 and 20000 iterations, respectively

a)

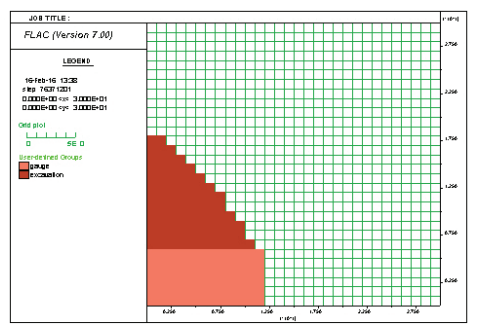

b)

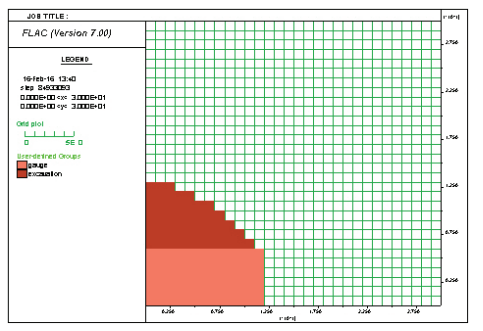

c)

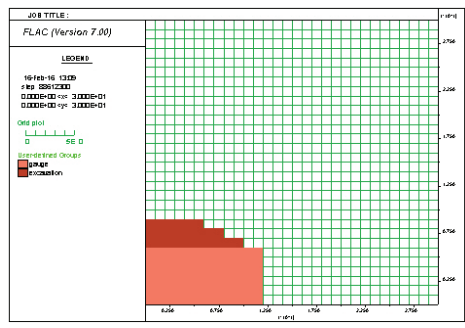

d)

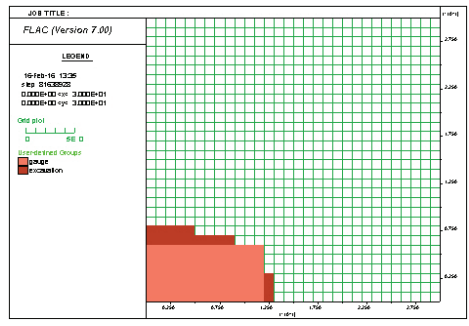

e)

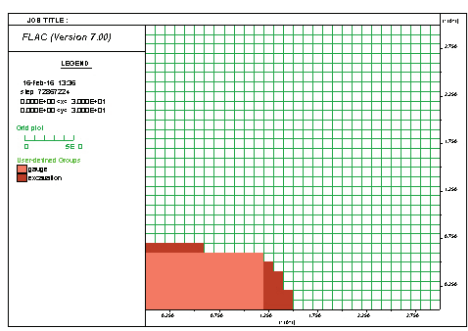

Fig. 4. Optimal shapes of excavation: (a) $p_{x} / p_{z}=1 / 4$, (b) $p_{x} / p_{z}=1 / 2$, (c) $p_{x} / p_{z}=1$, (d) $p_{x} / p_{z}=2$, (e) $p_{x} / p_{z}=4$ 


\section{SUMMARY AND CONCLUSIONS}

A shape optimization of underground excavation by simulated annealing is presented. Unlike other theoretical approaches the one considered in the paper takes into consideration a practical requirement of preserving fixed clearance gauge. The procedure utilized in the paper takes advantage of the one proposed by the authors in [4]. A condition of preserving clearance gauge is added. Furthermore, the algorithm is modified so that the excavation cross-section area may vary. Two types of shape changes are alternately imposed, tested and then accepted or rejected according to proper conditions. Finally, the optimal shape understood as proper material distribution over the FV grid may be obtained.

A showcase example of finding optimal excavation shape for five different in situ stress states was worked out using numerical computation. The results clearly show that:

- shapes regarded as optimal circumscribe the clearance gauge and have smooth contours,

- if vertical virgin stress is greater than horizontal one, then a crown arc has greater curvature and sides are almost straight; vertical size of the opening is significantly greater than gauge vertical size,

- if horizontal virgin stress is greater than vertical, then the sides are arched and the crown curvature is small; horizontal size of the opening is significantly greater than the gauge horizontal size.

The results obtained suggest applicability of the methodology introduced. The investigation will be for sure continued by the authors. For instance, refining mesh or using unstructured grid would result in obtaining smoother shapes giving more detailed information about optimal topology. Furthermore, multi-objective optimization, with various cost functions, should be considered as a further development of the research.

\section{REFERENCES}

[1] KAWA M., RÓŻAŃSKi A., SOBÓTKA M., A verification of shape optimization procedures of tunnel underground excavations, Górnictwo i Geoinżynieria, 2011, Book 2, 535-541, (in Polish).
[2] Ren G., Smith J.V., Tang J.W., XIE Y.M., Underground excavation shape optimization using an evolutionary procedure, Computers and Geotechnics, 2005, No. 32, $122-132$

[3] RÓŻAŃSKi A., SoBÓTKA M., A procedure of underground excavations shape optimization, Górnictwo i Geoinżynieria, 2009, Book 1, 519-529, (in Polish).

[4] SoвÓTKA M., ŁYDŻBA D., RÓŻAŃSKI A., Shape optimization of underground excavation by simulated annealing, Studia Geotechnica et Mechanica, 2013, 35(1), 209-218.

[5] NguYen T., GHABraie K., TrAn-CONG T., Simultaneous pattern and size optimisation of rock bolts for underground excavations, Computers and Geotechnics, 2015, 66, 264-277.

[6] SAlustowicz A., Zarys mechaniki górotworu, Wydawnictwo Śląsk, Katowice, 1968.

[7] XIE Y.M., Steven G.P., A simple evolutionary procedure for structural optimization, Comput. Struct., 1993, No. 49(5), 885-896.

[8] XIE Y.M., STEven G.P., Evolutionary structural optimization, Springer, Berlin, 1997.

[9] KirkPatrick S., Gelatt C., Vecchi M., Optimization by simulated annealing, Science, 1983, Vol. 220, No. 4598, 671-680.

[10] Sonmez F.O., Shape optimization of $2 D$ structures using simulated annealing, Computer Methods in Applied Mechanics and Engineering, 2007, 196, 35, 3279-3299.

[11] SовÓткA M., ŁydżBA D., Shape Optimization of Soil-steel Structure by Simulated Annealing, Procedia Engineering, 91, 304-309.

[12] RÓŻAŃSKI A., ŁYDŻBA D., JABŁOŃSKI P., Numerical study of the size of representative volume element for linear elasticity problem, Studia Geotechnica et Mechanica, 2013, 35(2), 6781.

[13] RÓŻAŃSKI A., ŁYDŻBA D., From digital image of microstructure to the size of representative volume element: B4C/Al composite, Studia Geotechnica et Mechanica, 2011, 33(1), 55-68.

[14] Chapman D., Metje N., StÄrk A., Introduction to tunnel construction. 2010, Vol. 3. CRC Press.

[15] RABCEWICZ L.V., Bemessung von Hohlraumbauten, die "Neue Österreichische Bauweise" und ihr Einfluß auf Gebirgsdruckwirkungen und Dimensionierung, Felsmechanik und Ingenieurgeologie, 1963, 1, 3-4.

[16] SHIM P.Y., MANOOCHEHRI S., Generating optimal configurations in structural design using simulated annealing, International Journal for Numerical Methods in Engineering, 1997, 40(6), 1053-1069.

[17] Metropolis N. et.al., Equation of state calculation by fast computing machines, The Journal of Chemical Physics, 1953, Vol. 21, No. 6, 1087-1092.

[18] FLAC Fast Lagrangian Analysis of Continua, User's Guide, Itasca Consulting Group Inc. Minneapolis, 2011.

[19] MajCherCZYK T., NiedBALSKi Z., KowAlSki M., 3D numerical modeling of road tunnel stability - The Laliki project, Archives of Mining Sciences, 2012, 57(1), 61-78. 